\section{A Diagnostic Challenge in COVID-19 Pandemic: PCP or COVID-19}

Sir,

The coronavirus disease 2019 (COVID-19) was declared as a pandemic in March, 2020. Its clinical presentation ranges from asymptomatic disease, to mild upper respiratory tract infection, to viral pneumonia, to respiratory failure, or even death. ${ }^{1}$ About $80 \%$ of patients have a mild or moderate disease, $14 \%$ severe and $5 \%$ critical. ${ }^{2}$ Pneumocystis carinii pneumonia (PCP) is an opportunistic infection and it usually infects immunocompromised individuals, or patients with advanced human immunodeficiency virus (HIV) infection. Symptoms include non-productive or dry cough, fever, shortness of breath, bilateral interstitial infiltrates, with or without hypoxemia. ${ }^{3}$

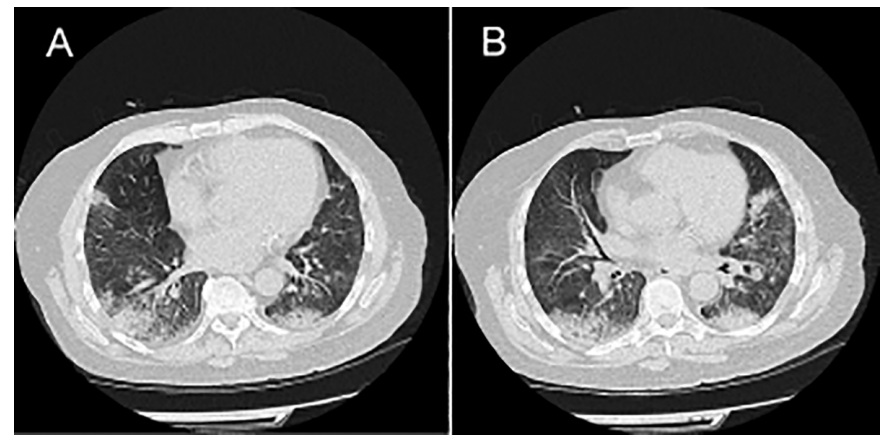

Figure 1 (A, B): CTChest showing bilateral pulmonary infiltrates.

A 67-year gentleman presented with shortness of breath, fever and cough. The background history was significant for diabetes and hypertension. Baseline imaging and routine blood tests were consistent with respiratory tract infection. The patient was clinically deteriorating, inflammatory markers were on the upward trend, and blood gases showed type 1 respiratory failure. He was admitted to the intensive care unit, and owing to severe hypoxemia, was intubated and subjected to mechanical ventilation. Treatment with broad-spectrum intravenous (IV) antibiotics was initiated; but, no clinical or radiological improvement was observed. A CT chest showed bilateral middle-lower lung patchy opacities / consolidations and interstitial pneumonia (Figure $1 \mathrm{~A}$ and B). On clinical suspicion and repeated COVID-19 negative swabs, negative blood and urine cultures, PCP treatment was initiated. There was an excellent clinical improvement. The patient was extubated and was subsequently weaned off oxygen. His HIV status was negative, but sputum and PCR showed PCP.

Pneumocystis jirovecii, the new name of pneumocystis carinii, usually causes pneumonia in immunocompromised patients and is considered an acquired immunodeficiency syndrome (AIDS)-defining condition. Due to wide spread use of highly active antiretroviral therapy (HAART), this infection has dramatically decreased in HIV positive individuals. In contrast, PCP is growing among HIV-negative patients, ${ }^{4}$ due to increasing use of immunosuppressive agents and corticosteroids for treatment of various underlying conditions. ${ }^{5}$ In this case, the presentation was of typical respiratory tract infection; and as per protocol in this pandemic, the top differential was a COVID-19 infection; but upon repeated COVID-19 negative swabs, and clinical deterioration, suspicion of PCP was made and appropriate treatment was started. There were no risk factors for PCP.

In conclusion, PCP can be a diagnostic challenge, especially in this COVID-19 pandemic. The clinical presentation can mimic a COVID-19 infection. It is even more challenging when there are no risk factors for PCP infection like immunosuppression or HIV infection.

\section{CONFLICT OF INTEREST:}

Authors declared no conflict of interest.

\section{AUTHORS' CONTRIBUTION:}

MMS, SY, SU: Conception and writing of the manuscript.

\section{REFERENCES}

1. Wang D, Hu B, Hu C, Zhu F, Liu X, Zhang J, et al. Clinical characteristics of 138 hospitalised patients with 2019 novel coronavirus-infected pneumonia in Wuhan, China. Jama 2020; 323(11):1061-9. doi: 10.1001/jama.2020.1585.

2. Wu Z, McGoogan JM. Characteristics of and important lessons from the coronavirus disease 2019 (COVID-19) outbreak in China: Summary of a report of 72314 cases from the chinese center for disease control and prevention. Jama 2020; 323(13):1239-42. doi: 10.1001/jama.2020. 2648.

3. Wilkin A, Feinberg J. Pneumocystis carnii pneumonia: A clinical review. American family Physician 1999; 60(6): 1699.

4. Martin SI, Fishman JA. AST infectious diseases community of practice. Pneumocystis pneumonia in solid organ transplantation. American J Transplant 2013; 13(s4):272-9. doi: 10.1111/ctr.13587.

5. Carmona EM, Limper AH. Update on the diagnosis and treatment of Pneumocystis pneumonia. Ther Adv Respir Dis 2011; 5(1):41-59. doi: 10.1177/1753465810380102.

Muhammad Mohsin Sajjad, Sidra Yousaf and Sajjad Ullah

Cavan General Hospital, RCSI Group, Ireland

Correspondence to: Dr. Muhammad Mohsin Sajjad, Cavan General Hospital, RCSI Group, Ireland

E-mail: drmohsin34567@gmail.com

Received: June 24, 2020; Revised: September 26, 2020; Accepted: October 01, 2020

DOI: https://doi.org/10.29271/jcpsp.2020.JCPSPCR.CR144 